\title{
Lobaplatin inhibits breast cancer progression, cell proliferation while it induces cell apoptosis by downregulating MTDH expression
}

This article was published in the following Dove Press journal:

Drug Design, Development and Therapy

Wuguo Tian
Shuai Hao
Bo Gao
Yan Jiang
Xiaohua Zhang
Shu Zhang
Lingji Guo
Jianjie Zhao
Gang Zhang
Yi Chen
Zhirong Li
Donglin Luo
Department of Breast, Thyroid
Surgery, Research Institute of Surgery,
Daping Hospital, Army Military
Medical University, Chongqing 400042,
China
Department of Breast, Thyroid Surgery, Research Institute of Surgery, Daping Hospital, Third Military Medical University, No.2 Chongqing Road 10, Chongqing 400042, China

Email luodonglin_12@I26.com
Correspondence: Donglin Luo

Tel +86236875 7950

Objective: Lobaplatin shows antitumor activity against a wide range of tumors, including metastatic breast cancer (BCa). The overexpression of metadherin (MTDH) is associated with poor prognosis of $\mathrm{BCa}$ patients. This study was designed to investigate the effect of lobaplatin on MCF-7 cell proliferation and its association with MTDH expression.

Patients and methods: Clinical treatment for BCa using lobaplatin, in combination with other general chemotherapy drugs, was administered to $32 \mathrm{BCa}$ patients. The safety, effectiveness, and prognosis in lobaplatin-treated BCa patients were compared with those in controls $(n=32)$. In vitro experiments were performed in MCF-7 cells to investigate the effect of lobaplatin on cell proliferation, apoptosis, and MTDH expression.

Results: We found the intraoperative local chemotherapy using lobaplatin was safe and effective for $\mathrm{BCa}$ treatment, in comparison with the patients administered general chemotherapy drugs. Treatment of MCF-7 cell cultures with lobaplatin significantly reduced cell proliferation and increased cell apoptotic percentage. The expression of MTDH and Bcl-2 was inhibited by lobaplatin and that of Bax was increased by lobaplatin. Moreover, we observed the inhibition of MTDH by shRNA reduced cell proliferation and enhanced cell apoptosis.

Conclusion: Lobaplatin was a safe and effective adjuvant chemotherapy for BCa. The effect of lobaplatin on inhibiting MCF-7 cell proliferation and inducing cell apoptosis might be, as least in part, mediated by suppressing the expression of oncogene MTDH.

Keywords: breast cancer, lobaplatin, proliferation, apoptosis, MTDH

\section{Introduction}

Breast cancer $(\mathrm{BCa})$ is a common malignancy among women, with an increasing prevalence worldwide. ${ }^{1,2} \mathrm{BCa}$-related death is the second cause of cancer death among women worldwide. ${ }^{1}$ The drug and chemoradiotherapy resistance, higher recurrence during follow-up, and higher rates of genetic mutations in $\mathrm{BCa}$ patients make $\mathrm{BCa}$ treatment challenging. ${ }^{1,3,4}$ It is well known that the rate of $\mathrm{BCa}$ cells' resistance to chemoradiotherapy is high. ${ }^{5,6}$ These obstacles make it an urgent need to find new agents or neoadjuvant chemotherapy for treatment of $\mathrm{BCa}$.

Lobaplatin is a representative of the third-generation platinum antineoplastic agents, which has wide-range activities of overcoming tumor resistance to chemoradiotherapy drugs, including cisplatin and carboplatin. ${ }^{1,7,8}$ Studies have shown the antitumor activity of lobaplatin in cancers, including human cholangiocarcinoma, ${ }^{9,10}$ lung cancer, ${ }^{11}$ human cervical cancer, ${ }^{12}$ melanoma, ${ }^{13}$ gastric cancer, ${ }^{7,14}$ esophageal squamous cell carcinoma, ${ }^{15}$ and $\mathrm{BCa} .{ }^{16-18}$ Some clinical studies reported that the intraoperative local chemotherapy using lobaplatin for BCa was safe and effective, ${ }^{17}$ while others 
reported that administration of lobaplatin as a neoadjuvant chemotherapy to docetaxel and epirubicin regimen for triplenegative BCa (TNBC) showed increased side effects. ${ }^{15-17,19}$ Regimen using lobaplatin for TNBC, primary and metastatic $\mathrm{BC}$ had been reported. ${ }^{16-18}$ It has been reported that lobaplatin inhibited cancer cell proliferation and induced cancer cell apoptosis by arresting cell cycle progression, thus leading to the suppression of cancer metastasis and development of antitumor activity. ${ }^{11-13,15}$

Metadherin (MTDH) is an oncogenic protein and functions by promoting cancer cell proliferation, invasion, and drug resistance. ${ }^{20,21}$ The expression of MTDH was associated with various signaling pathways, including AKT signaling pathway, and miRNAs which were involved in cell proliferation and tumorigenesis. ${ }^{22-26}$ The downregulation of $\mathrm{MTDH}$, however, could induce the apoptosis of BCa MCF-7 cells, ${ }^{1}$ prostate cancer DU145 cells, ${ }^{26}$ and lung cancer A549 cells. ${ }^{23}$ Wang showed that cell proliferation and the expression of MTDH in lobaplatin-treated MCF-7 cells were inhibited, with increased cell apoptosis (in Chinese). ${ }^{27}$ Similarly, Chen showed that intraoperative local chemotherapy using lobaplatin in radical mastectomy for BCa resulted in reduced exfoliated cancer cells. ${ }^{17}$ Engel et al reported that the administration of lobaplatin inhibited $\mathrm{BCa}$ cell proliferation. ${ }^{28} \mathrm{In}$ addition, the downregulation of MTDH in MCF-7 cells was related to cell apoptosis. ${ }^{1}$ These studies might suggest that lobaplatin treatment for cancer cells and inhibition of MTDH were, respectively, associated with the inhibition of cancer cell proliferation. However, little information is available on $\mathrm{MTDH}$ expression in response to lobaplatin treatment for $\mathrm{BCa}$.

To investigate the effect of lobaplatin on $\mathrm{BCa}$ and to explore the association of MTDH expression with lobaplatininduced cell apoptosis, we performed the clinical casecontrol study using lobaplatin as an intraoperative local chemotherapy for BCa. Cellular experiments were performed to detect the influence of lobaplatin on MCF-7 cell proliferation and MTDH expression. The association between lobaplatin and MTDH expression would be discussed. This study would provide us with more basic information on the relation of $\mathrm{MTDH}$ expression with lobaplatin in $\mathrm{MCF}-7$ cells in vitro.

\section{Patients and methods}

\section{Subjects, treatments, and surgical procedure}

Female patients with primary diagnosis of BCa were enrolled in this study from Daping Hospital, Army Military Medical
University, Chongqing, China, between March 2009 and September 2012. Patients were diagnosed with BCa by imaging (magnetic resonance imaging) and pathology. All BCa patients had Karnofsky Performance Score $\geq 80$. Subjects participating in this study met the following criteria: 1) no obvious chemotherapy taboo; 2) no obvious dysfunction in heart, lung, liver, and kidney; 3) no significant difference in basic information between patients when randomly assigned; and 4) no history of malignancy and diabetes. Patients were assigned to control or lobaplatin-treated (experimental) group according to individual willingness. A total of 32 patients were assigned to the experimental group $(n=32)$ and the other 32 age-matched patients were assigned to the control group $(n=32)$. Experimental protocols were approved by the Ethics Committee of Daping Hospital. Informed written consents were obtained from these 64 patients with $\mathrm{BCa}$.

All patients were subjected to radical mastectomies. After cleaning, the wounds were washed with normal saline (NS) supplemented with and without lobaplatin $(50 \mathrm{mg}$ in $100 \mathrm{~mL}$ NS). Before closing them with stitches, $50 \mathrm{mg}$ lobaplatin (in $100 \mathrm{~mL} \mathrm{NS}$ ) was sprayed onto the surface of tumor bed, axillary wound cavity, and other areas suspicious for having residual cancer cells. ${ }^{17}$ The total volume of drainage fluids in 72 hours, wound healing time, complications (local pain, nausea, vomiting, diarrhea), myelosuppression (white cell count and platelet count), and liver and renal functions (creatinine and alanine aminotransferase) were recorded and compared. All patients received normal chemoradiotherapy plus lobaplatin as intraoperative local chemotherapy.

At 72 hours post-surgery, drainage fluid was collected, centrifuged, and cell pellets were collected for smears, which were then subjected to the detection of exfoliated cancer cells. ${ }^{17}$ Also, recurrences and other complications during the follow-up of 4 years were compared.

\section{Cells, culture conditions, and lobaplatin treatment}

Human BCa cell line MCF-7 was obtained from ATCC (Manassas, VA, USA) and cultured in DMEM (Thermo Fisher Scientific, Waltham, MA, USA) supplemented with $10 \%$ fetal bovine serum (Thermo Fisher Scientific) and $1 \%$ antibiotic-antimycotic (Thermo Fisher Scientific) at $37^{\circ} \mathrm{C}$ with $5 \% \mathrm{CO}_{2}$. For lobaplatin treatment, MCF-7 cells were treated with $0,5,10$, and $50 \mathrm{mg} / \mathrm{L}$ lobaplatin (Hainan Chang'an International Pharmaceutical Co., Ltd, Hainan, China) for 24 hours. ${ }^{7}$ Each cellular experiment was performed in triplicate. 


\section{Cell transfection}

To generate an MTDH-low-expressing cell model in vitro, we transfected MCF-7 cells with shRNA-MTDH plasmids (based on pSUPER-EGFP vector; OligoEngine, Seattle, WA, USA) using Lipofectamine 2000 (Thermo Fisher Scientific) according to the manufacturer's instructions. ${ }^{29}$ Cells were transfected with shRNA-MTDH and pSuper-EGFP vector containing control shRNAs (shRNA-NC) for 6 hours prior to lobaplatin treatment.

\section{Cell proliferation assay}

The effect of lobaplatin and MTDH inhibition on MCF-7 cells viability was examined using the MTT assay (Thermo Fisher Scientific). Briefly, cells were plated into 96-well plates $\left(5 \times 10^{4}\right.$ cells/well) and treated with lobaplatin for 24,48 , and 72 hours. Then, the cell cultures were treated with MTT reagent for 4 hours and dimethyl sulfoxide $(150 \mu \mathrm{L} /$ well) for another 20 minutes, followed by detection at $\mathrm{OD} 570 \mathrm{~nm}$.

\section{Cell apoptosis analysis by flow cytometry}

To confirm the effect of lobaplatin and MTDH expression on cell apoptosis, the MCF- 7 cells $\left(1 \times 10^{5}\right.$ cells/well in six-well plates) were treated with either shRNA-MTDH transfection or lobaplatin for 24 hours. Then, Annexin V-FITC Apoptosis Detection Kit (Sigma) was utilized to measure cell apoptosis. The percentage of apoptotic cells was examined using a FACS Canto ${ }^{\mathrm{TM}}$ II cytometry instrument and analyzed using CellQuest software (BD Diagnostics, Franklin lakes, NJ, USA).

\section{RNA isolation and real-time polymerase chain reaction}

Total RNA was extracted from MCF-7 cells using an RNeasy kit (Qiagen, Valencia, CA, USA) at 24 hours post-lobaplatin treatment, and cDNA was generated using the M-MLV1 reverse transcription kit (Takara, Tokyo, Japan). Amplification was conducted on PE-9600 thermal cycler (Thermo Fisher Scientific) using a SYBR Green PCR kit (Qiagen, Los Angeles, CA, USA) with the following reaction conditions: $95^{\circ} \mathrm{C}$ for 5 minutes, 40 cycles of $95^{\circ} \mathrm{C}$ for 30 seconds, and $60^{\circ} \mathrm{C}$ for 30 seconds. The $2^{-\Delta \Delta C t}$ methods were used for calculating the mRNA relative expression level of MTDH gene, with normalization to $\beta$-actin.

\section{Western blot analysis}

Protein was isolated from MCF-7 cells using RIPA lysis buffer (Thermo Fisher Scientific), followed by quantification using a BCA kit (Qiagen). Protein was separated onto sodium dodecyl sulfate-polyacrylamide gel electrophoresis and electroblotted onto polyvinylidene difluoride membranes (EMD Millipore, Billerica, MA, USA) according to standard methods. Membranes were then incubated with specific primary antibody anti-MTDH (Sigma), anti-Bax (Cell Signaling Technology [CST], Danvers, MA, USA), anti-Bcl-2 (CST), and anti- $\beta$-actin (CST) at $4^{\circ} \mathrm{C}$ overnight and related secondary antibodies at room temperature for 40 minutes. Bio-Rad Quantity One software (Bio-Rad) was used to analyze protein expression with the enhanced chemiluminescence system.

\section{Statistical analysis}

Continuous variables are expressed as mean \pm SD. Statistical significances between groups were assessed using Student's $t$-test, and those among groups were assessed using one-way analysis of variance followed by Dunnett's post hoc test. Categorical variables are expressed as frequencies, and differences between groups were compared by using chi-squared statistics. $P<0.05$ was considered statistically significant.

\section{Results \\ Multi-point spraying of lobaplatin promotes wound healing and reduces recurrence}

As shown in Table 1, there was difference in the volume of drainage within 72 hours after operation between the two groups $(P<0.05)$. The white cell count, platelet count, creatinine, alanine aminotransferase, wound healing time, and complications between groups were similar ( $P>0.05$; Table 1$)$. The detection rate of exfoliated cancer cells in the control group was higher than that in the treatment group $(P<0.05)$. Within 72 hours postoperations, exfoliated cancer cells were found in the drainage of four patients $(4 / 32,12.50 \%)$ treated with lobaplatin, which was obviously lower than that in the control group $(10 / 32,31.25 \% ; P<0.05)$. No differences were found in the rates of adverse events (recurrence and metastasis) between the groups during a 4-year follow-up. These data suggested the safety of using lobaplatin as intraoperative local chemotherapy for $\mathrm{BCa}$ patients.

\section{Lobaplatin suppresses MCF-7 cells proliferation and promotes cell apoptosis}

To investigate the effect of lobaplatin on $\mathrm{BCa}$ cell proliferation, we treated MCF-7 cells in vitro with lobaplatin and found inhibited cell viability (Figure 1A). In comparison with the control cells, lobaplatin-treated MCF-7 cells showed inhibited cell viability $(P<0.05)$. MCF-7 cells treated with 
Table I The differences in general data and complications within 72 hours after operations, and the adverse events during a 4-year follow-up

\begin{tabular}{|c|c|c|c|}
\hline Items & Control & Treatment & $P$-value \\
\hline \multicolumn{4}{|c|}{ General data before and within $\mathbf{7 2}$ hours after operations } \\
\hline Age (years) & $46.4 \pm 5.7$ & $47.1 \pm 6.3$ & $>0.05$ \\
\hline WBC $\left(\times 10^{9}\right)$ & $7.61 \pm 2.01$ & $7.32 \pm 1.20$ & $>0.05$ \\
\hline $\operatorname{PLT}\left(\times 10^{9}\right)$ & $229.40 \pm 74.27$ & $227.12 \pm 45.37$ & $>0.05$ \\
\hline Creatinine $(\mu \mathrm{mol} / \mathrm{L})$ & $76.8 I \pm 5.44$ & $75.43 \pm 6.37$ & $>0.05$ \\
\hline $\mathrm{ALT}(\mathrm{U} / \mathrm{L})$ & $33.67 \pm 4.76$ & $33.18 \pm 6.01$ & $>0.05$ \\
\hline Drainage $(\mathrm{mL})$ & $572.15 \pm 47.23$ & $504.81 \pm 60.48$ & $<0.05$ \\
\hline Healing time (days) & $18.2 \pm 1.1$ & $17.7 \pm 2.7$ & $>0.05$ \\
\hline \multicolumn{4}{|c|}{ Complications within 72 hours after operations } \\
\hline Pain & $34.75 \%(11 / 32)$ & $31.25 \%(10 / 32)$ & $>0.05$ \\
\hline Nausea & $25.00 \%(8 / 32)$ & $28.13 \%(9 / 32)$ & $>0.05$ \\
\hline Vomiting & $12.50 \%(4 / 32)$ & $12.50 \%(4 / 32)$ & $>0.05$ \\
\hline Diarrhea & $6.25 \%(2 / 32)$ & $3.13 \%(1 / 32)$ & $>0.05$ \\
\hline Patients with exfoliated cancer cells & $12.50 \%(4 / 32)$ & $31.25 \%(10 / 32)$ & $<0.05$ \\
\hline \multicolumn{4}{|c|}{ Adverse events during follow-up ( 4 years) } \\
\hline Recurrence & $3.13 \%(1 / 32)$ & $6.25 \%(2 / 32)$ & $>0.05$ \\
\hline Metastasis & $0(0 / 32)$ & $3.13 \%(1 / 32)$ & $>0.05$ \\
\hline
\end{tabular}

Note: Patients in treatment group were treated with lobaplatin after cleaning procedures, followed by radical mastectomies for BCa patients. Abbreviations: ALT, alanine aminotransferase; BCa, breast cancer; PLT, platelet count; WBC, white cell count.

$5 \mathrm{mg} / \mathrm{L}$ lobaplatin presented with significantly reduced cell viability compared with controls $(P<0.05)$, which was then enhanced with the increment of lobaplatin concentration. In addition, flow cytometry analysis showed that lobaplatin promoted cell apoptosis in a dose-dependent manner. After being treated with lobaplatin for 24 hours, $13.20 \% \pm 1.22 \%$, $16.73 \% \pm 2.10 \%$, and $34.0 \% \pm 1.35 \%$ apoptotic cells were found in MCF-7 cells treated with $5 \mathrm{mg} / \mathrm{L}(P<0.05), 10 \mathrm{mg} / \mathrm{L}$ $(P<0.01)$, and $50 \mathrm{mg} / \mathrm{L}$ lobaplatin $(P<0.01)$, respectively, which were significantly higher than that of control cells $(4.22 \% \pm 0.12 \%$; Figure $1 \mathrm{Bi}$, ii). These data suggest that lobaplatin inhibited MCF-7 cell proliferation and induced cell apoptosis.

\section{Lobaplatin decreases $\mathrm{Bcl}-2 / \mathrm{Bax}$ ratio}

In addition, we found the expression of $\mathrm{Bcl}-2$ and $\mathrm{Bax}$ proteins was inhibited and promoted by lobaplatin, respectively (Figure 2A and B). The Bcl-2/Bax ratio in MCF-7 cells was decreased by lobaplatin administration $(<1.36 \pm 0.50)$ in a dose-dependent manner (Figure 2C) compared with that in control cells $(4.24 \pm 0.26, P<0.001)$. This fact suggested that lobaplatin inhibited the $\mathrm{Bcl}-2 / \mathrm{Bax}$ ratio in MCF-7 cells.

\section{Lobaplatin suppresses MTDH expression in MCF-7 cells}

It was reported previously that MTDH expression was associated with cancer cells' proliferation and the latter could be dysregulated by lobaplatin. ${ }^{1,7,13,29}$ Accordingly, we thought there might be an association between MTDH and lobaplatinmediated apoptosis. As expected, we found the expression of MTDH mRNA and protein was reduced in MCF-7 cells receiving lobaplatin treatments, in a dose-dependent manner (Figure 3). This result revealed the expression of MTDH in MCF-7 cells was inhibited by lobaplatin administration.

\section{MTDH inhibition promotes MCF-7 cell apoptosis}

Since the administration of lobaplatin suppressed MTDH expression and promoted the apoptosis of MCF-7 cells, we thought the inhibition of MTDH might be a crucial regulator for lobaplatin-mediated cell apoptosis. To investigate this, we knocked out MTDH expression in MCF-7 cells by shRNA technology (Figure 4A). We found the knockdown of MTDH expression significantly increased the percentage of apoptotic MCF-7 cells $(45.20 \% \pm 1.35 \%)$ compared with control ( $4.51 \% \pm 0.12 \%, P<0.001$; Figure $4 \mathrm{~B}$ ), and was superior to $50 \mathrm{mg} / \mathrm{L}$ lobaplatin administration $(28.00 \% \pm 2.12 \%$, $P<0.001)$.

Moreover, we also found the knockdown of MTDH showed superior effect on suppressing Bcl-2 expression $(P<0.01$, Figure $4 \mathrm{C})$ and inducing Bax expression $(P<0.05$, Figure $4 \mathrm{D})$ to $50 \mathrm{mg} / \mathrm{L}$ lobaplatin administration in MCF-7 cells. Accordingly, prominent lower Bcl-2/Bax ratio $(0.44 \pm 0.18)$ was observed in shRNA-MTDH groups compared with that in $50 \mathrm{mg} / \mathrm{L}$ lobaplatin-administered cells $(1.04 \pm 0.23, P<0.01$; Figure 4E). These data demonstrate that 

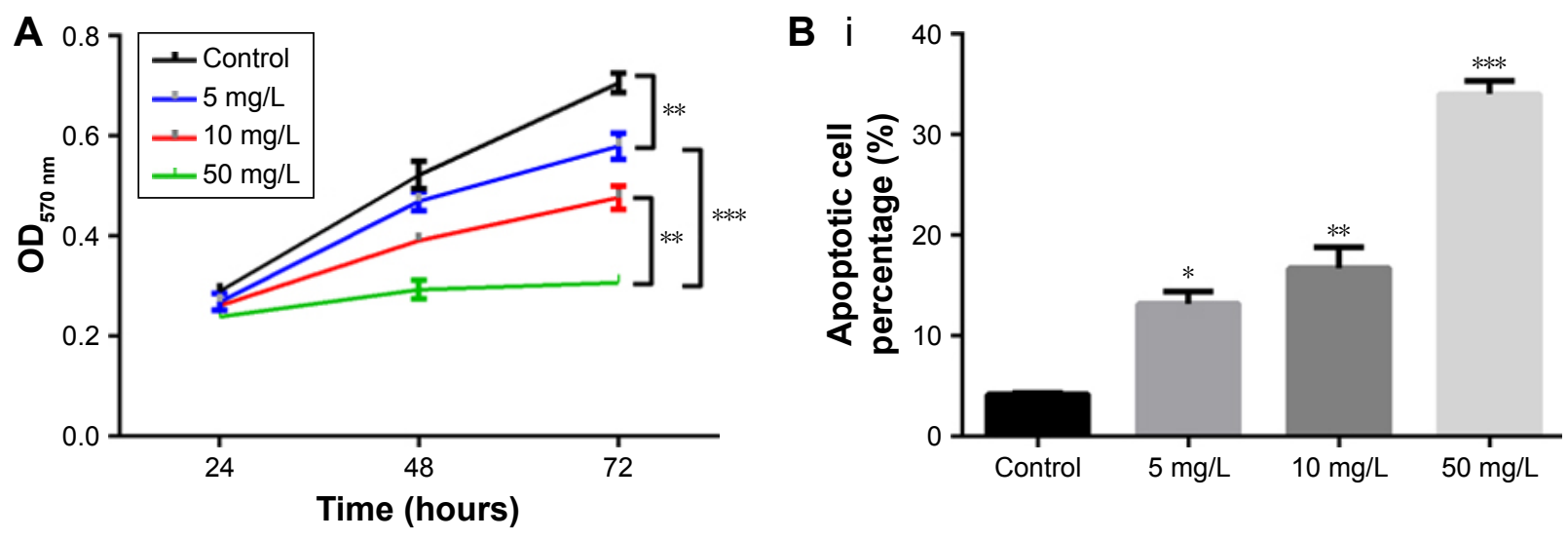

B ii

Control
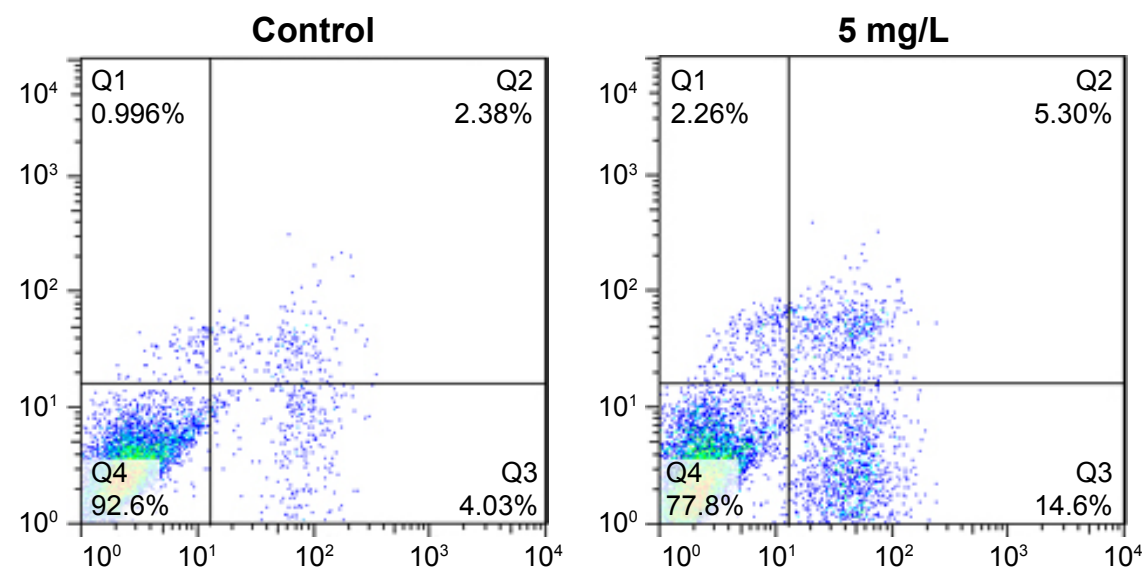

$10 \mathrm{mg} / \mathrm{L}$
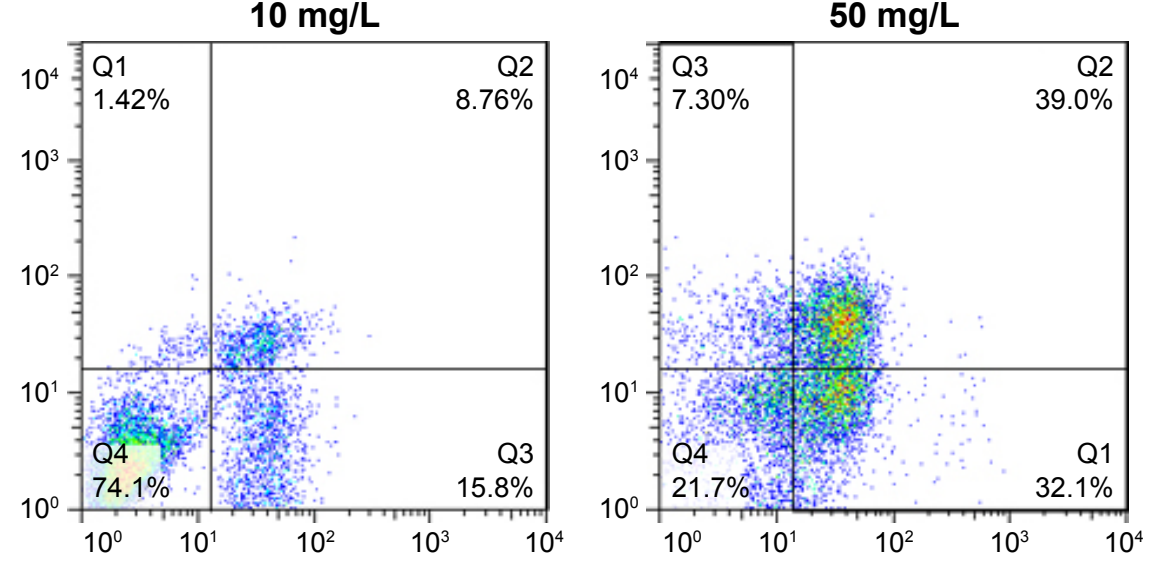

Figure I Lobaplatin induces MCF-7 cell apoptosis.

Notes: (A) Cell proliferation analysis. ( $\mathbf{B i}$ and ii) Cell apoptosis analysis using flow cytometric analysis. $*$, $* *$, and $* * *$ indicate $\mathbf{P}<0.05,0.0 \mathrm{I}$, and $0.00 \mathrm{I}$ vs control, respectively.

lower MTDH expression correlated with higher apoptotic percentage in MCF-7 cells, and lobaplatin-mediated MCF-7 cell apoptosis was at least, in part, related with inhibition of MTDH and Bcl-2/Bax ratio.

\section{Discussion}

Lobaplatin is an effective chemotherapy agent with antitumor characteristics. ${ }^{1,13,17}$ The overexpression and inhibition of MTDH promoted and inhibited cancer cell proliferation, respectively. ${ }^{1,29}$ There are plenty of in vitro and in vivo clinical studies showing the antitumor effect of lobaplatin. ${ }^{12,13,18}$ Our current data suggested that lobaplatin-reduced cell proliferation of MCF-7 cells was related to the inhibition of an oncogene MTDH.

Using the case-control study, we demonstrated the safety and efficacy of using lobaplatin as one of the chemotherapy 
A

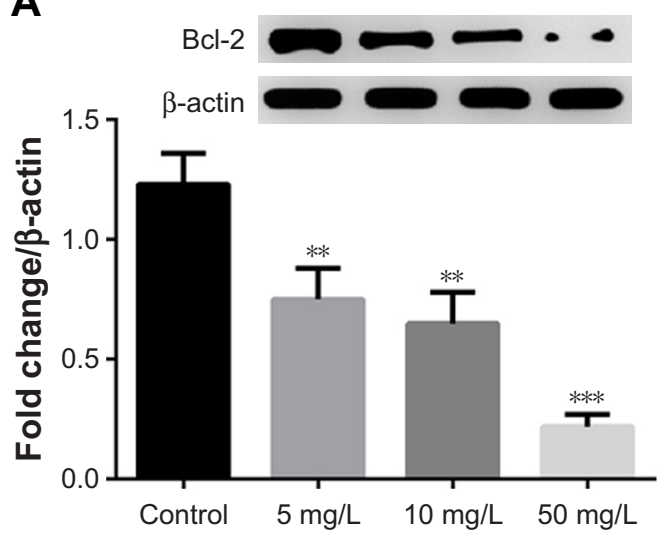

B

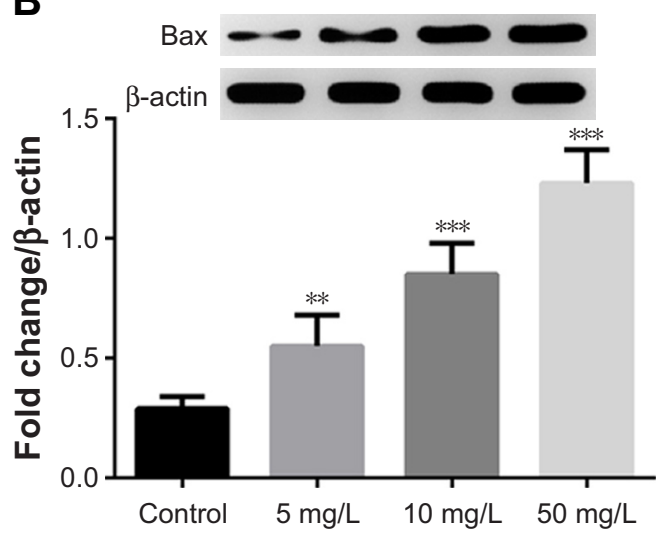

C

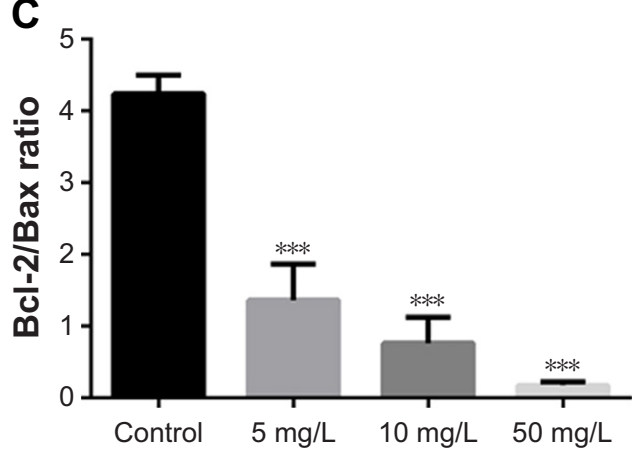

Figure 2 Lobaplatin affected the expression of MTDH and apoptosis-related protein in MCF-7 cells.

Notes: $(\mathbf{A}$ and $\mathbf{B})$ The protein expression of $\mathrm{Bcl}-2$ and Bax by Western blot analysis. (C) The Bcl-2/Bax ratio. **, and *** indicate $P<0.05,0.0 \mathrm{I}$, and $0.00 \mathrm{I}$ vs control, respectively.

Abbreviation: MTDH, metadherin.

agents for $\mathrm{BCa}$ treatment after surgery. These results were in accordance with a previous clinical study by $\mathrm{Chen} .{ }^{17} \mathrm{BCa}$ patients who were treated with general agents combined with lobaplatin after radical mastectomies showed higher amount of drainage and lower exfoliated cancer cells compared with patients receiving only general agents. In our study, the fact that no differences were observed in complications within 72 hours after operations and during a 4-year follow-up revealed the safety of using lobaplatin for $\mathrm{BCa}$ treatment. This was in consistent with the results from Chen et al. This suggests the effectiveness of using lobaplatin. However, $\mathrm{Wu}$ et al showed that although the addition of lobaplatin as neoadjuvant chemotherapy to TNBC treatments improved the pathologic complete response, the rate of side effects (anemia and thrombocytopenia) in $\mathrm{BCa}$ patients receiving lobaplatin was elevated. ${ }^{16}$ All these studies suggested the effectiveness
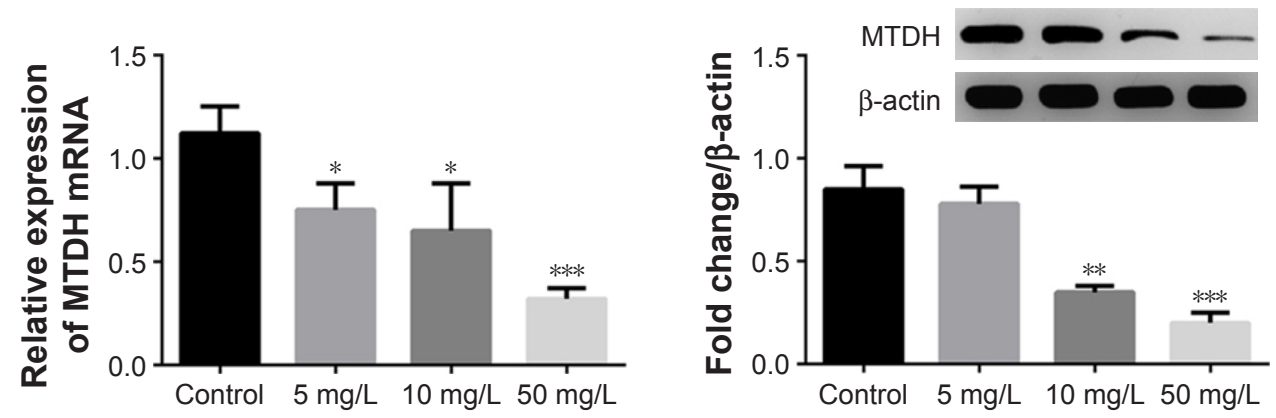

Figure 3 The expression of MTDH in lobaplatin-treated cells.

Notes: The mRNA expression level (left) and protein fold change of MTDH (right), respectively, were detected using qRT-PCR and Western blot analysis. *, **, and *** indicate $P<0.05,0.01$, and 0.001 vs control, respectively.

Abbreviations: MTDH, metadherin; qRT-PCR, quantitative reverse transcriptase-polymerase chain reaction. 
A
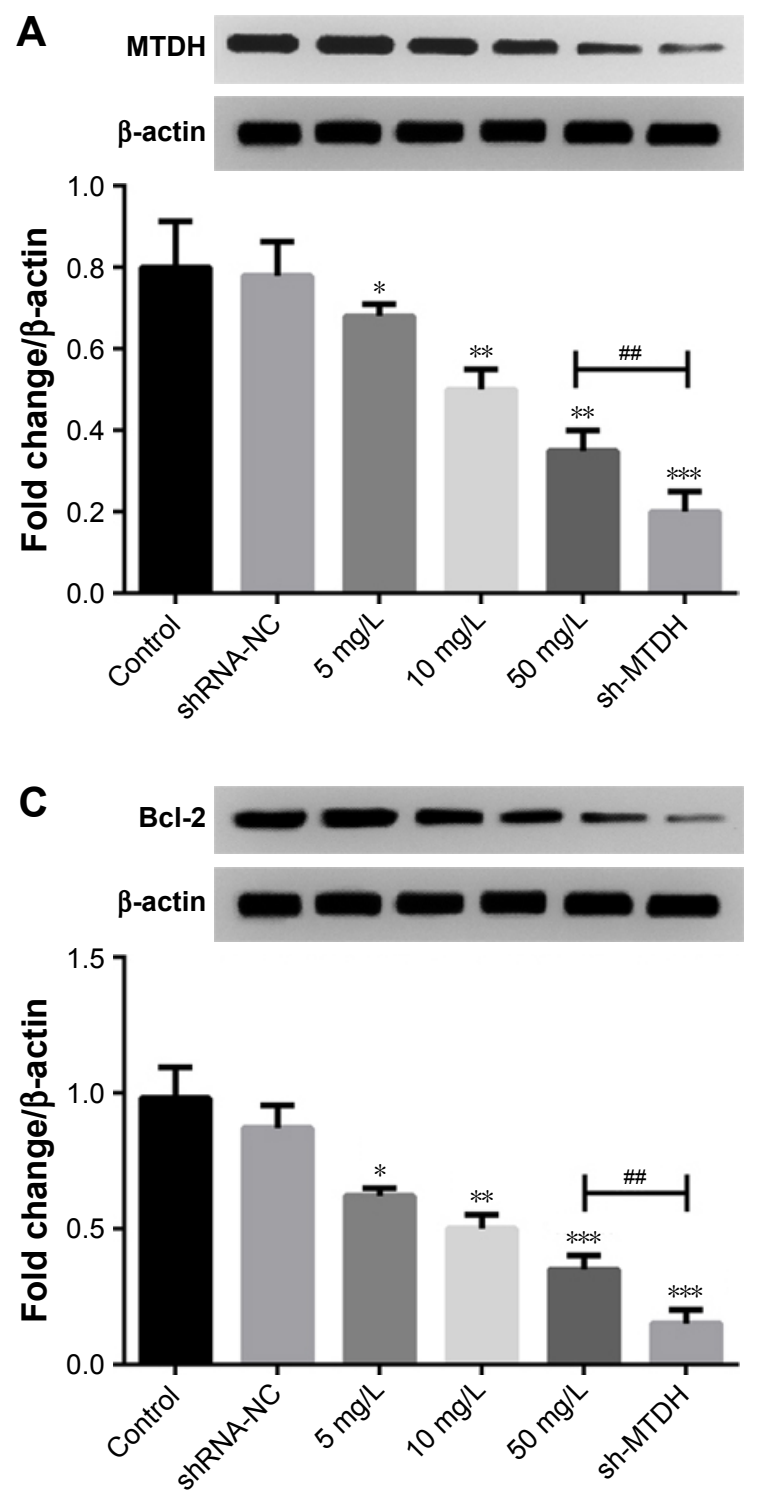

B

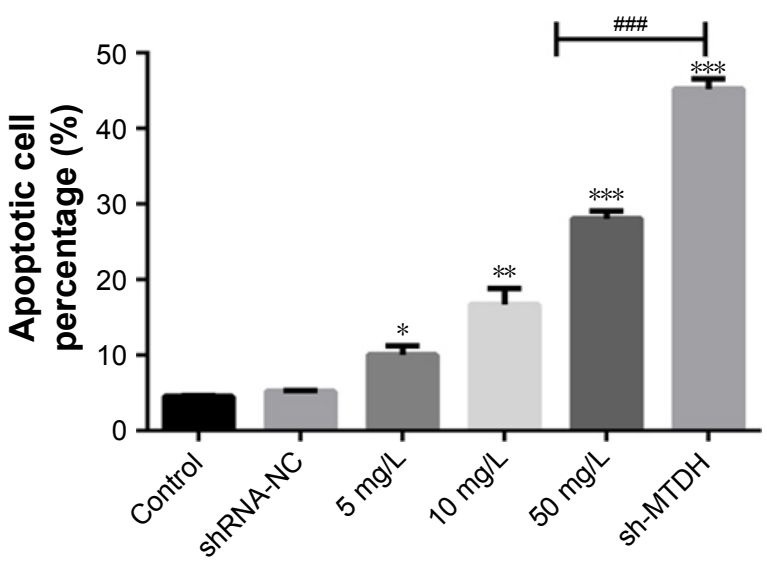

D
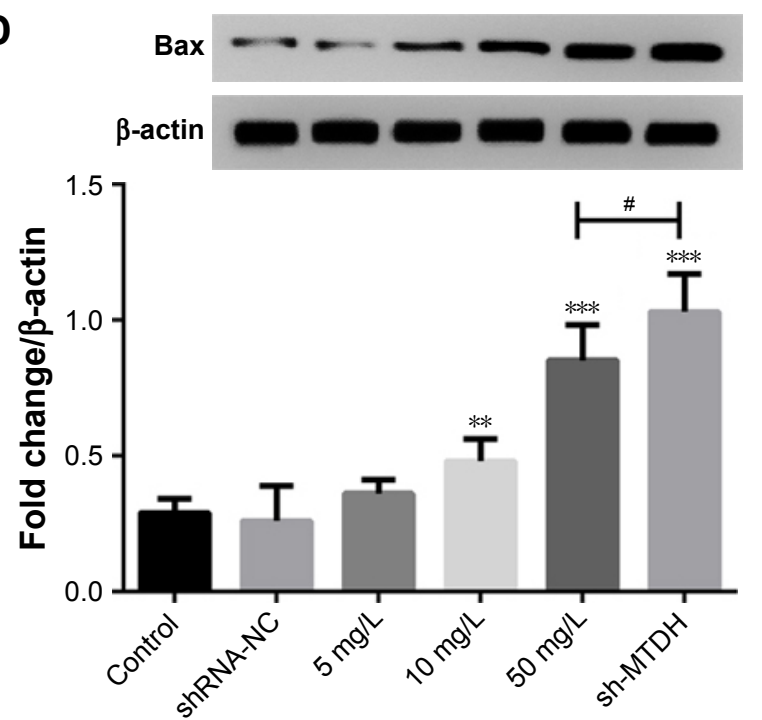

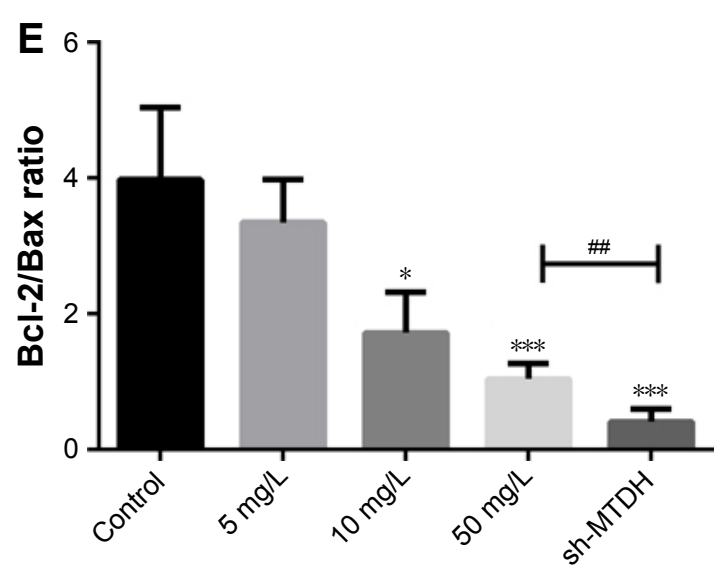

Figure 4 Effect of MTDH inhibition on MCF-7 cell apoptosis and proliferation.

Notes: (A) The expression of MTDH protein by Western blot analysis. (B) Cell apoptosis was analyzed using flow cytometric analysis. (C and $\mathbf{D}$ ) The expression of Bcl-2 and Bax proteins, respectively. (E) The Bcl-2/Bax ratio. *, **, and *** indicate $P<0.05,0.01$, and $0.00 \mathrm{I}$ vs control, respectively. \#, ${ }^{\#}$, and ${ }^{\# \#}$ indicate differences at $P<0.05$, $0.0 \mathrm{I}$, and $0.00 \mathrm{I}$ vs $50 \mathrm{mg} / \mathrm{L}$ lobaplatin.

Abbreviation: $\mathrm{MTDH}$, metadherin. 
of using lobaplatin for BCa treatment. But the opposing point on side effects is worth pursuing further.

Yang et al showed that lobaplatin arrested B16-F10 melanoma cell cycle progression and induced apoptosis, thus inhibiting the migration and invasion of melanoma cells. ${ }^{13}$ Similar results of using lobaplatin treatment for in vitro cancers had been reported on human cervical cancer cells, ${ }^{14}$ gastric carcinoma cells, ${ }^{12}$ and esophageal squamous cell carcinoma cells. ${ }^{15}$ The antiapoptotic protein Bcl-2 and the proapoptotic protein Bax are associated with cell apoptosis and cancer cell resistance. ${ }^{13,15,30}$ The Bcl-2/Bax ratio, particularly, could be used as a marker for therapeutic response to radiotherapy. ${ }^{31,32} \mathrm{Du}$ et al showed that lobaplatin induced esophageal squamous cell carcinoma KYSE-410 and EC-109 cells' apoptosis and decreased the Bcl-2/Bax ratio. ${ }^{42}$ In our in vitro experiments, we demonstrated that the administration of lobaplatin to $\mathrm{MCF}-7$ cells significantly reduced cell proliferation, Bcl-2/Bax ratio, and induced cell apoptosis. Collectively, these data suggest that lobaplatin might be an effective chemotherapeutic agent in BCa treatment.

Previous studies had shown that MTDH played important roles in tumorigenesis. ${ }^{20,29,33}$ The evidence on the association of MTDH with microRNAs, AKT, and $\beta$-catenin signaling pathways reveals the crucial functional roles of MTDH overexpression in tumorigenesis..$^{21,24,25,34,35} \mathrm{Li}$ et al showed that MTDH was a direct target of tumor suppressor miRNA miR-30a-5p and the overexpression of miR-30a-5p induced hepatocellular carcinoma cell apoptosis via inhibiting MTDH, upregulating PTEN protein, and suppressing Akt. ${ }^{35}$ The expression of MTDH was regulated by various tumor suppressing and oncogenic miRNAs. ${ }^{35-37}$ The expression of MTDH in tumors was associated with tumor progression via regulating epithelial-mesenchymal transition and chemoresistance in cancer cells, ${ }^{29,38,39}$ suggesting the crucial roles of MTDH expression in tumorigenesis and progression.

In $\mathrm{BCa}$, the overexpression of MTDH was associated with an aggressive phenotype and short overall survival as well as short distant metastasis-free survival. ${ }^{20}$ In addition, the downregulation of a tumor suppressing miRNA, miR-26a, was associated with the overexpression of MTDH in TNBC, and the ectopic expression of miR-26a suppressed MTDH and the subsequent cell proliferation and metastasis. ${ }^{21}$ Our data suggest that lobaplatin induces MCF-7 cells proliferation as well as MTDH suppression. Also, the inhibition of MTDH by shRNA in MCF-7 cells showed similar results to lobaplatin treatment. Taken together, we thought there might be an association between MTDH inhibition and lobaplatin-induced cell apoptosis in $\mathrm{BCa}$.

MTDH is involved in various pathways $s^{21,24,25,40}$. It has been reported that MTDH could promote the differentiation of cancer cell stemness in BCa cells by activating Twist1, thus promoting tumorigenicity. ${ }^{41}$ A latest study suggested that the expression of MTDH in BCa cells could be decreased by the overexpression of tumor suppressor F-box and WD-40 domain protein 7 (FBXW7, hCDC4), thus inhibiting cancer cell proliferation and promoting apoptosis. ${ }^{33}$ In our present study, we suggest that lobaplatin-induced cell apoptosis in $\mathrm{BCa}$ cells might be associated with MTDH inhibition. Also, MTDH-shRNA-induced apoptosis in MCF-7 cells was at least, in part, related to increase in Bcl-2/Bax ratio. These suggest that MTDH plays important roles in tumorigenicity, and the MTDH-mediated mechanism in BCa progression should be explored in future experiments.

\section{Conclusion}

In summary, we primarily found treatment of $\mathrm{BCa}$ patients with lobaplatin was a safe and effective strategy. In the in vitro experiments, we found lobaplatin promoted MCF-7 cell apoptosis, inhibited cell proliferation, Bcl-2/Bax ratio, as well as the expression of MTDH in BCa cells. We found that lobaplatin-mediated MCF-7 cell apoptosis was at least, in part, mediated by suppression of the oncogene MTDH.

\section{Acknowledgment}

This study was supported by the Chongqing Science and Technology Commission Huimin Plan-funded projects (cstc2016kjhmpt100-16).

\section{Disclosure}

The authors report no conflicts of interest in this work.

\section{References}

1. Luo Z, Hu X, Xiong H, et al. A polysaccharide from Huaier induced apoptosis in MCF-7 breast cancer cells via down-regulation of MTDH protein. Carbohydr Polym. 2016;151:1027-1033.

2. Desantis CE, Fedewa SA, Goding Sauer A, Kramer JL, Smith RA, Jemal A. Breast cancer statistics, 2015: convergence of incidence rates between black and white women. CA Cancer J Clin. 2016;66(1):31-42.

3. Garcia-Murillas I, Schiavon G, Weigelt B, et al. Mutation tracking in circulating tumor DNA predicts relapse in early breast cancer. Sci Transl Med. 2015;7(302):302ra133.

4. Rheinbay E, Parasuraman P, Grimsby J, et al. Recurrent and functional regulatory mutations in breast cancer. Nature. 2017;547(7661):55-60.

5. Li J, Liu J, Guo N, Zhang X. Reversal of multidrug resistance in breast cancer MCF-7/ADR cells by h-R3-siMDR1-PAMAM complexes. Int $J$ Pharm. 2016;511(1):436-445.

6. Liu J, Li J, Liu N, et al. In vitro studies of phospholipid-modified PAMAM-siMDR1 complexes for the reversal of multidrug resistance in human breast cancer cells. Int J Pharm. 2017;530(1-2):291-299.

7. Yin CY, Lin XL, Tian L, Ye M, Yang XY, Xiao XY. Lobaplatin inhibits growth of gastric cancer cells by inducing apoptosis. World $J$ Gastroenterol. 2014;20(46):17426.

8. Dai HY, Liu L, Qin SK, He XM, Li SY. Lobaplatin suppresses proliferation and induces apoptosis in the human colorectal carcinoma cell Line LOVO in vitro. Biomed Pharmacother. 2011;65(3):137-141. 
9. Wang Z, Tang X, Zhang Y, et al. Lobaplatin induces apoptosis and arrests cell cycle progression in human cholangiocarcinoma cell line RBE. Biomed Pharmacother. 2012;66(3):161-166.

10. Lieke T, Ramackers W, Bergmann S, Klempnauer J, Winkler M, Klose J. Impact of salinomycin on human cholangiocarcinoma: induction of apoptosis and impairment of tumor cell proliferation in vitro. BMC Cancer. 2012;12(1):466.

11. Xie CY, Xu YP, Jin W, Lou LG. Antitumor activity of lobaplatin alone or in combination with antitubulin agents in non-small-cell lung cancer Anticancer Drugs. 2012;23(7):698-705.

12. Li X, Ran L, Fang W, Wang D. Lobaplatin arrests cell cycle progression, induces apoptosis and alters the proteome in human cervical cancer cell line CaSki. Biomed Pharmacother. 2014;68(3):291-297.

13. Yang F, Yu Y, Lei Q, et al. Lobaplatin arrests cell cycle progression, induces apoptosis and impairs migration and invasion in B16-F10 melanoma cell line in vitro. Biomed Pharmacother. 2015;69:402-408.

14. Li Y, Liu B, Yang F, et al. Lobaplatin induces BGC-823 human gastric carcinoma cell apoptosis via ROS-mitochondrial apoptotic pathway and impairs cell migration and invasion. Biomed Pharmacother. 2016;83: 1239-1246.

15. Du L, Fei Z, Song S, Wei N. Antitumor activity of lobaplatin against esophageal squamous cell carcinoma through caspase-dependent apoptosis and increasing the $\mathrm{Bax} / \mathrm{Bcl}-2$ ratio. Biomed Pharmacother 2017;95:447-452.

16. Wu X, Tang P, Li S, et al. A randomized and open-label Phase II trial reports the efficacy of neoadjuvant lobaplatin in breast cancer. Nat Commun. 2018;9(1):832.

17. Chen WX. Clinical observation of intraoperative local chemotherapy with lobaplatin in breast cancer modified radical mastectomy. Int J Clin Exp Med. 2017;10(7):10834-10839.

18. Wang Z, Xu L, Wang H, et al. Lobaplatin-based regimens outperform cisplatin for metastatic breast cancer after anthracyclines and taxanes treatment. Saudi J Biol Sci. 2018;25(5):909-916.

19. Li D. A clinical study of combination regimen containing lobaplatin in treating advanced metastatic breast cancer. Journal of Shanxi Medical College for Continuing Education. 2016;4:004.

20. Tokunaga E, Nakashima Y, Yamashita N, et al. Overexpression of metadherin/MTDH is associated with an aggressive phenotype and a poor prognosis in invasive breast cancer. Breast Cancer. 2014;21(3):341-349.

21. Liu P, Tang H, Chen B, et al. miR-26a suppresses tumour proliferation and metastasis by targeting metadherin in triple negative breast cancer. Cancer Lett. 2015;357(1):384-392.

22. Li PP, Yao QM, Zhou H, et al. Metadherin contribute to BCR signaling in chronic lymphocytic leukemia. Int J Clin Exp Pathol. 2014; 7(4): 1588

23. Zou Y, Qin X, Xiong H, Zhu F, Chen T, Wu H. Apoptosis of human non-small-cell lung cancer A549 cells triggered by evodiamine through MTDH-dependent signaling pathway. Tumour Biol. 2015;36(7): 5187-5193.

24. Liang C, Ding J, Yang Y, Deng L, Li X. MicroRNA-433 inhibits cervical cancer progression by directly targeting metadherin to regulate the AKT and $\beta$-catenin signalling pathways. Oncol Rep. 2017;38(6): 3639-3649.

25. Yu C, Liu Y, Tan H, et al. Metadherin regulates metastasis of squamous cell carcinoma of the head and neck via AKT signalling pathwaymediated epithelial-mesenchymal transition. Cancer Lett. 2014; 343(2):258-267.

Drug Design, Development and Therapy

\section{Publish your work in this journal}

Drug Design, Development and Therapy is an international, peerreviewed open-access journal that spans the spectrum of drug design and development through to clinical applications. Clinical outcomes, patient safety, and programs for the development and effective, safe, and sustained use of medicines are the features of the journal, which
26. Qian B, Yao Y, Liu C, Zhang J, Chen H, Li H. SU6668 modulates prostate cancer progression by downregulating MTDH/AKT signaling pathway. Int J Oncol. 2017;50(5):1601-1611.

27. Wang Q. Effect of Lobaplatin on Apoptosis of Breast Cancer MCF-7 Cells and Expression of MTDH Gene [master's thesis]. The Fourth Military Medical University (in Chinese); 2010.

28. Engel JB, Martens T, Hahne JC, et al. Effects of lobaplatin as a single agent and in combination with TRAIL on the growth of triple-negative p53-mutated breast cancers in vitro. Anticancer Drugs. 2012;23(4):426-436.

29. Meng X, Brachova P, Yang S, et al. Knockdown of MTDH sensitizes endometrial cancer cells to cell death induction by death receptor ligand TRAIL and HDAC inhibitor LBH589 co-treatment. PLoS One. 2011;6(6):e20920.

30. Sharifi S, Barar J, Hejazi MS, Samadi N. Roles of the Bcl-2/Bax ratio, caspase- 8 and 9 in resistance of breast cancer cells to paclitaxel. Asian Pac J Cancer Prev. 2014;15(20):8617-8622.

31. Mackey TJ, Borkowski A, Amin P, Jacobs SC, Kyprianou N. Bcl-2/Bax ratio as a predictive marker for therapeutic response to radiotherapy in patients with prostate cancer. Urology. 1998;52(6):1085-1090.

32. Khodapasand E, Jafarzadeh N, Farrokhi F, Kamalidehghan B, Houshmand $\mathrm{M}$. Is $\mathrm{Bax} / \mathrm{Bcl}-2$ ratio considered as a prognostic marker with age and tumor location in colorectal cancer? Iran Biomed J. 2015; 19(2):69-75.

33. Chen X, Li XY, Long M, et al. The FBXW7 tumor suppressor inhibits breast cancer proliferation and promotes apoptosis by targeting MTDH for degradation. Neoplasma. 2018;65(2):201-209.

34. Zhu L, Zhang P, Yang Y, et al. Abstract A53: metadherin functions as a laminin receptor that is essential for metastasis and is associated with poor survival in osteosarcoma. Cancer Res. 2014; 74(20 Supplement):A53.

35. Li WF, Dai H, Ou Q, Zuo GQ, Liu CA. Overexpression of microRNA30a-5p inhibits liver cancer cell proliferation and induces apoptosis by targeting MTDH/PTEN/AKT pathway. Tumour Biol. 2016; 37(5):5885-5895.

36. Tang Y, Liu X, Su B, et al. MicroRNA-22 acts as a metastasis suppressor by targeting metadherin in gastric cancer. Mol Med Rep. 2015; 11(1):454-460.

37. Wang Y, Wei Y, Tong H, et al. MiR-302c-3p suppresses invasion and proliferation of glioma cells via down-regulating metadherin (MTDH) expression. Cancer Biol Ther. 2015;16(9):1308-1315.

38. Wang Z, Tang ZY, Yin Z, et al. Metadherin regulates epithelial-mesenchymal transition in carcinoma. Onco Targets Ther. 2016;9(Issue 1): 2429-2436.

39. Li W, Zhai L, Zhao C, Lv S. MiR-153 inhibits epithelial-mesenchymal transition by targeting metadherin in human breast cancer. Breast Cancer Res Treat. 2015;150(3):501-509.

40. Li PP, Feng LL, Chen N, et al. Metadherin contributes to the pathogenesis of chronic lymphocytic leukemia partially through Wnt/ $\beta$-catenin pathway. Med Oncol. 2015;32(2):21.

41. Liang Y, Hu J, Li J, et al. Epigenetic activation of TWIST1 by MTDH promotes cancer stem-like cell traits in breast cancer. Cancer Res. 2015; 75(17):3672-3680.

42. Du L, Fei Z, Wei N, et al. Antitumor activity of Lobaplatin against esophageal squamous cell carcinoma through caspase-dependent apoptosis and increasing the Bax/Bcl-2 ratio. Biomed Pharmacother. 2017;95:447-452.

\section{Dovepress}

has also been accepted for indexing on PubMed Central. The manuscript management system is completely online and includes a very quick and fair peer-review system, which is all easy to use. Visit http://www.dovepress.com/testimonials.php to read real quotes from published authors. 